\title{
Estabelecimento de Ciclo de Cura de Pré-Impregnados Aeronáuticos
}

\author{
Michelle L. Costa, Mirabel C.Rezende \\ Divisão de Materiais, IAE, CTA \\ Edson C. Botelho \\ Divisão de Materiais, IAE, CTA \\ Departamento de Materiais e Tecnologia, UNESP
}

Resumo: Os compósitos poliméricos podem ser produzidos via moldagem em autoclave, onde as condições de processamento podem ser otimizadas a partir do conhecimento físico-químico da matriz polimérica. A evolução da cinética da reação de cura ocorre simultaneamente com as modificações no comportamento reológico do sistema polimérico, sendo comum denominar o fenômeno de comportamento reo-cinético. O presente trabalho tem como objetivo conhecer os parâmetros de cura, cinéticos e reológicos, de três diferentes sistemas de pré-impregnados de resina epóxi (cura a $177^{\circ} \mathrm{C}$ ), conhecidos como F161, F584 e 8552, hoje usados na indústria aeronáutica brasileira. Este estudo foi realizado com o auxílio das técnicas de DSC e reologia, utilizando-se análises dinâmicas e isotérmicas. Com isso, foi possível estabelecer a ordem de reação e a cinética de cura dos sistemas estudados. Neste estudo, foram utilizados como modelos matemáticos o de ordem n e o autocatalítico com ordem total de aproximadamente 2 . A temperatura de gel foi de $\sim 100{ }^{\circ} \mathrm{C}$, e o tempo de gel correspondente foi de 135 segundos. A partir do conhecimento da cinética de cura e dos parâmetros reológicos dos sistemas de pré-impregnados foi possível estabelecer um ciclo de cura destinado à consolidação das peças aeronáuticas via moldagem em autoclave.

Palavras-Chave: Ciclo de cura, pré-impregnado, cinética de cura, análises térmicas e reológicas.

\section{Establishment of Cure Cycle of Aeronautic Prepregs}

Abstract: Autoclave molding produces polymer composites, where the processing conditions can be optimized with physicochemical knowledge of the polymeric matrix. The cure reaction evolves simultaneously with changes in rheology, which is normally refered to as rheo-kinetic behavior. With the knowledge of the appropriate cure cycle one can identify the steps in which pressure should be applied and when to raise the temperature. This paper is aimed at investigating the cure, kinetics and rheological parameters of three prepreg epoxy systems, namely F161, F584 and 8552, which are currently used in the Brazilian aeronautic industry. Differential scanning calorimetry (DSC) and rheological techniques have been used, both in the isothermal and dynamic modes. Kinetics data were obtained from dynamic and isothermal DSC, with rheological measurements being carried out during the cure. The mathematical models used were the nth order reaction model and the autocatalytic model with order of 2 . The gel temperature was $\sim 100{ }^{\circ} \mathrm{C}$, and the corresponding gelification time was $135 \mathrm{~s}$. With the determination of the kinetics for the cure and of the rheological parameters of the prepreg systems, a cure cycle should be established that led to high-performance composites using the autoclave molding.

Keywords: Cure cycle, prepreg, cure kinetics, thermal and rheological analyses.

\section{Introdução}

A combinação de baixa massa específica com valores elevados de resistência e rigidez que os compósitos poliméricos avançados proporcionam, permite que essa classe de materiais seja empregada nas indústrias aeronáutica e espacial em substituição aos materiais metálicos tradicionais ${ }^{[1-6]}$. Componentes estruturais de aeronaves para fins civis e militares, tais como: flapes, leme, carenagens, empenagens, naceles, aileron, tanques de combustível, profundor, cone de cauda entre outros, que anteriormente eram fabricados em ligas de alumínio, titânio e aços especiais, estão hoje sendo fabricados na sua grande maioria em estruturas laminadas de materiais pré-impregnados (prepregs) de compósitos poliméricos avançados ${ }^{[7-10]}$. Os compósitos poliméricos avançados podem oferecer aos componentes estruturais elevada resistência à fadiga e à corrosão, baixa condutividade térmica, isolamentos acústico e térmico e facilidade de obtenção

Autor para correspondência: Mirabel C. Rezende, Divisão de Materiais, IAE, CTA, Praça Marechal do Ar Eduardo Gomes 50 - Vila das Acácias, CEP. 12228-901, São José dos Campos, SP. E-mail: mirabel@iae.cta.br 
de geometrias complexas, assegurando-lhes melhor desempenho em serviço ${ }^{[10-12]}$.

Com a constante evolução tecnológica que o processamento e as matérias-primas precursoras dos compósitos poliméricos avançados vêm apresentando, novos espaços em aplicações não aeronáuticas estão sendo conquistados contribuindo, simultaneamente, para o crescimento do interesse industrial em desenvolver e produzir produtos mais leves com elevados padrões da qualidade e de resistência estrutural ${ }^{[13-19]}$. Um desenvolvimento bem sucedido é obtido quando se equilibra a eficácia do método de fabricação, com a redução do seu custo e o elevado padrão da qualidade do produto. Neste caso, maior atenção deve ser dada aos métodos de fabricação automatizados, à escolha de matérias-primas adequadas e aos procedimentos de manipulação dos materiais, pois esses garantem as grandes escalas de produção exigidas nos outros setores não aeronáuticos ${ }^{[14]}$.

O termo pré-impregnado é aplicado a todo produto intermediário, pronto para moldagem, e pode ser definido como sendo uma composição de fibras de reforço com um determinado polímero, termorrígido formulado ou termoplástico, em uma particular fração em massa ${ }^{[3-5]}$. Esses materiais oferecem uma combinação de consistência e processabilidade de produto, devido à quantidade controlada de resina, porém requerem a aplicação de vácuo e pressão durante o processo de cura. As duas matérias-primas básicas para a produção de pré-impregnados são a matriz polimérica e as fibras de reforço na forma de tecido ou fitas unidirecionais. A fibra de carbono é um dos reforços que possui maior módulo e resistência disponível para aplicação estrutural. Já a resina epóxi termorrígida utilizada no pré-impregnado apresenta-se parcialmente polimerizada, estágio de cura conhecido como estágio $\mathrm{B}$, exigindo o seu armazenamento a $-18^{\circ} \mathrm{C}$ para a desaceleração do processo de polimerização ${ }^{[3-13]}$. Historicamente na produção da indústria aeronáutica, a maioria dos componentes é fabricada pelo método convencional de corte e laminação manual das camadas do material pré-impregnado, sobre um molde devidamente preparado com agente desmoldante, seguido da cura em autoclave $\mathrm{e}^{[5]}$.

Para obter a porcentagem volumétrica das fibras e garantir a união entre as camadas, os laminados são curados em autoclave de acordo com o ciclo de cura sugerido pelo fabricante dos pré-impregnados (temperatura e pressão controladas), combinada com a ação de vácuo para garantir alta resistência, ótimo acabamento superficial e uma distribuição uniforme da resina no compósito ${ }^{[3,5-7]}$.

Toda a atividade na autoclave é monitorada e avaliada por manômetros, termopares e vacuômetros. Com o aquecimento ocorrem mudanças no estado físico da resina, sendo a mais importante a que ocorre na região de gel. Ultrapassada essa região, o fluxo da resina através do laminado é prejudicado, pois essa já se encontra na região viscoelástica ${ }^{[3,5]}$. Logo, a aplicação da pressão deve ser feita antes dessa região, para permitir uma molhabilidade adequada das fibras e promover a compactação do laminado, caracterizando um ciclo de cura ótimo ${ }^{[6,7,9-13]}$. Após ter sido completada a cura, todos os laminados são desmoldados e inspecionados tanto visualmente como pelo uso da técnica de ultrasom.

Apesar das vantagens que os materiais compósitos apresentam e que motivam a sua utilização na indústria aeroespacial, essa classe de materiais tem como desvantagem, em comparação aos metais, a susceptibilidade aos danos, perdendo muito de sua integridade estrutural quando isto ocorre. Os danos podem ocorrer durante o processamento da matéria-prima, fabricação da peça, manuseio, transporte, armazenagem, manutenção ou em serviço ${ }^{[3,14-18]}$.

Com o objetivo de minimizar as perdas em componentes termorrígidos, hoje de difícil reaproveitamento, devido às características da matriz termorrígida e dos reforços, estudos têm sido realizados para entender a tecnologia de processamento de materiais compósitos. O domínio dessa tecnologia está diretamente ligado à compreensão da química das resinas, utilizadas na impregnação dos reforços, e de seus prováveis mecanismos de cura; à obtenção das propriedades mecânicas finais desejadas, via escolha correta dos pré-impregnados e dos ciclos de cura mais adequados.

Assim, o presente trabalho tem como objetivo conhecer os parâmetros de cura, cinéticos e reológicos, de três diferentes sistemas de pré-impregnados de resina epóxi, conhecidos como F161, F584 e 8552, hoje usados na indústria aeronáutica brasileira. Este estudo foi realizado com o auxílio das técnicas de DSC e reologia, utilizando-se análises dinâmicas e isotérmicas. Com isso, foi possível estabelecer a ordem de reação e a cinética de cura dos sistemas estudados. Além disso, foi possível mostrar que um único ciclo de cura $\left(177^{\circ} \mathrm{C}\right)$ é capaz de curar todos os três tipos de pré-impregnados apresentando economia para a indústria aeronáutica com a otimização do uso da autoclave.

\section{Materiais e Métodos}

\section{Materiais}

Neste trabalho foram utilizados três tipos de pré-impregnados: um constituído de tecido de fibra de carbono tipo 8 HS (Harness-Satin) impregnado com o sistema de resina epóxi F584, outro com mesmo tipo de reforço só que impregnado com resina epóxi modificada com termoplástico conhecida como 8552 e por último, um tecido de fibra de vidro impregnado com resina epóxi tipo F161. Os dois primeiros materiais são utilizados em estruturas primárias, já o último é usado em estruturas secundárias de uma aeronave. Todos os préimpregnados são produzidos pela empresa Hexcel Composites e devem ser armazenados em temperaturas inferiores a $18{ }^{\circ} \mathrm{C}$ negativos, podendo permanecer estocado nesta condição de 6 a 12 meses. Os materiais estudados encontravam-se armazenados sob refrigeração adequada por 6 meses.

\section{Caracterização dos pré-impregnados}

As análises de calorimetria exploratória diferencial (DSC) foram realizadas em um equipamento Perkin-Elmer Modelo Pyris 1- DSC, previamente calibrado com índio e zinco, sob fluxo constante de nitrogênio $(20 \mathrm{~mL} / \mathrm{min})$ e razão de aque- 
cimento de $2,5^{\circ} \mathrm{C} / \mathrm{min}$. As amostras de pré-impregnados $(5,4 \mathrm{a}$ $5,7 \mathrm{mg}$ ) foram encapsuladas em um porta-amostra de alumínio do tipo selado. As variáveis relacionadas com a cinética de cura foram obtidas a partir de varreduras dinâmicas de 30 a $300{ }^{\circ} \mathrm{C}$ realizadas sob as razões de aquecimento de $2,5^{\circ} \mathrm{C} / \mathrm{min}$; $5,0{ }^{\circ} \mathrm{C} / \mathrm{min}$ e $10{ }^{\circ} \mathrm{C} / \mathrm{min}$, bem como a partir de isotermas.

As análises reológicas foram realizadas em amostras de pré-impregnados de fibras de carbono/epóxi com diâmetro de $25 \mathrm{~mm}$ e espessura de $0,3 \mathrm{~mm}$, utilizando-se um reômetro de tensão controlada da Rheometric Scientific, modelo SR5 , com pratos paralelos. Os ensaios foram realizados à freqüência constante de $1 \mathrm{rad} / \mathrm{s}$ e tensão de $500 \mathrm{~Pa}$ a diferentes razões de aquecimento: $2,5^{\circ} \mathrm{C} / \mathrm{min} ; 5^{\circ} \mathrm{C} / \mathrm{min} ; 10^{\circ} \mathrm{C} / \mathrm{min}$, para obtenção da temperatura de gel. Além disso, ensaios isotérmicos também foram realizados para a determinação do tempo de gelificação de cada sistema em estudo.

\section{Resultados e Discussão}

\section{Cinética das reações de cura}

Durante a cura de uma resina epóxi ocorrem mudanças na viscosidade do sistema, devido a um aumento na densificação das ligações cruzadas existentes neste termorrígido. Devido às reações ocorrerem em uma fase condensada, a taxa da reação de cura é controlada pela atividade dos grupos funcionais e pela mobilidade dos mesmos. Durante os primeiros estágios da reação, quando a taxa de movimentação dos grupos é maior que a taxa de colisões moleculares, a reação é controlada pela reatividade química dos grupos envolvidos na reação ${ }^{[20-22]}$. Conforme a reação prossegue, existe um aumento da ramificação das cadeias até que o sistema atinja a gelificação; que por sua vez causa um aumento na densificação das ligações cruzadas até que a mobilidade dos centros reativos seja progressivamente restringida e a reação começa a ser controlada pela difusão. Esse é o fator que limita o grau de conversão na temperatura de cura, $T_{c}$, o qual está abaixo da transição vítrea máxima do sistema de resina epóxi completamente curado, $\mathrm{Tg}_{\infty}$. Conforme o grau das ligações cruzadas aumenta, a Tg da epóxi também aumenta até atingir o valor de $T_{c}$, que é o ponto onde o sistema alcança o estado vítreo e se vitrifica. Nestas condições a cinética diminui e a extensão da conversão tende praticamente a valores limites constantes ${ }^{[22-25]}$.

No presente trabalho o estudo cinético da reação de cura dos sistemas de resina epóxi ficou restrito à primeira etapa da reação, ou seja, antes do controle da difusão. Para isto, modelos matemáticos simples foram adotados, tais como o modelo de cinética de ordem $n$ e de ordem autocatalítico (equações 1 e 2) ${ }^{[22-27]}$.

$$
\begin{aligned}
& \frac{d \alpha}{d t}=k(1-\alpha)^{n}, \text { ordem } \mathrm{n}, \\
& \frac{d \alpha}{d t}=k(1-\alpha)^{m} \alpha^{n}, \text { autocatalítico. }
\end{aligned}
$$

sendo: $\alpha$ o grau de conversão da reação química, $m$ e $n$ somados correspondem à ordem de reação, $k$ é a constante de velocidade e $d \alpha / d t$ é a primeira derivada de $\alpha$ em relação ao tempo.

O calor liberado na reação de cura está diretamente relacionado com o grau de conversão medido em determinada etapa do processo de cura. A técnica de DSC pode ser usada para medir a entalpia $(\Delta H)$ envolvida em qualquer reação em função do tempo, quando uma pequena quantidade de amostra é submetida a um programa de aquecimento dinâmico ou isotérmico. O calor total liberado para completar a reação de cura é igual à área sob a curva do calor gerado em função do tempo ou da temperatura. Os valores de $a$ são obtidos a partir dos valores das entalpias parciais, $\Delta H_{\text {parcial }}$ na tempertura (T) ou tempo (t), ou seja, como mostra a equação (3):

$$
\alpha=\frac{\Delta H_{\text {parcial }}}{\Delta H},
$$

onde $\Delta H$ é a entalpia total envolvida na reação.

Assim, de posse das equações descritas anteriormente e das varreduras dinâmicas no sistema de pré-impregnado de car-

Tabela 1. Parâmetros cinéticos obtidos a partir do método dinâmico para três razões de aquecimento: 2,$5 ; 5$ e $10^{\circ} \mathrm{C} / \mathrm{min}$ dos sistemas de pré-impregnados $F 584,8552$ e $F 161$.

\begin{tabular}{cccccccc}
\hline $\begin{array}{c}\text { Matriz do } \\
\text { pré-impregnado }\end{array}$ & $\begin{array}{c}\text { Razão aquecim. } \\
\left({ }^{\circ} \mathbf{C} / \mathbf{m i n}\right)\end{array}$ & $\begin{array}{c}\mathbf{T}_{\text {incial }} \\
\left({ }^{\circ} \mathbf{C}\right)\end{array}$ & $\begin{array}{c}\mathbf{T}_{\text {final }} \\
\left({ }^{\circ} \mathbf{C}\right)\end{array}$ & $\begin{array}{c}-\Delta \boldsymbol{H} \\
(\mathbf{J} / \mathbf{g})\end{array}$ & $\begin{array}{c}\boldsymbol{I n} \boldsymbol{A} \\
\left(\boldsymbol{s}^{-1}\right)\end{array}$ & $\begin{array}{c}\boldsymbol{E} \boldsymbol{a} \\
(\mathbf{k J / m o l})\end{array}$ & $\boldsymbol{n}$ \\
\hline \multirow{2}{*}{$\mathbf{F 5 8 4}$} & 2,5 & 140 & 242 & 560 & $7,9 \pm 0,7$ & $56,7 \pm 2,7$ & $1,4 \pm 0,1$ \\
& 5,0 & 145 & 240 & 554 & $9,9 \pm 0,4$ & $62,7 \pm 1,5$ & $1,3 \pm 0,1$ \\
& 10,0 & 163 & 244 & 557 & $11,9 \pm 0,5$ & $69,6 \pm 1,9$ & $1,1 \pm 0,1$ \\
\hline \multirow{2}{*}{$\mathbf{8 5 5 2}$} & 2,5 & 140 & 237 & 587 & $7,3 \pm 0,9$ & $54,6 \pm 2,2$ & $1,3 \pm 0,1$ \\
& 5,0 & 144 & 237 & 580 & $9,9 \pm 0,4$ & $62,4 \pm 1,8$ & $1,2 \pm 0,1$ \\
& 10,0 & 151 & 246 & 596 & $8,9 \pm 0,2$ & $58,4 \pm 0,8$ & $0,9 \pm 0,1$ \\
\hline \multirow{2}{*}{$\mathbf{F 1 6 1}$} & 2,5 & 122 & 165 & 52,7 & $18,7 \pm 0,9$ & $89,6 \pm 3,9$ & $1,5 \pm 0,1$ \\
& 5,0 & 140 & 196 & 49,9 & $18,8 \pm 0,4$ & $90,3 \pm 3,4$ & $1,2 \pm 0,1$ \\
& 10,0 & 155 & 208 & 49,6 & $25,6 \pm 0,2$ & $108,8 \pm 6,2$ & $1,1 \pm 0,1$ \\
\hline
\end{tabular}


bono/epóxi realizados, em diferentes razões de aquecimento, foi possível conhecer os parâmetros envolvidos na cinética de cura, através do método de Borchardt-Daniels ${ }^{[25-27]}$, como mostra a Tabela 1.

Na Tabela $1, \mathrm{~T}_{\text {inicial }}$ e $\mathrm{T}_{\text {final }}$ descrevem o intervalo de temperatura em que foi estudada a cinética de cura dos pré-impregnados, sempre mantendo o início do estudo em $5 \%$ e o final em $90 \%$ de conversão, uma vez que é sabido que no início da reação o processo é muito rápido e desordenado, sendo difícil estabelecer qualquer parâmetro cinético nesta fase, bem como no final da reação, no qual poucos grupamentos ainda estão por reagir e a reação passa a ser controlada pelo processo de difusão.

Com isso, observou-se que o início do evento exotérmico é registrado em temperaturas mais baixas quanto menor for a razão de aquecimento. Conseqüentemente, o calor e a ordem de reação aumentam com a redução da razão de aquecimento. Este comportamento pode estar relacionado com fatores instrumentais (deslocamento da temperatura para valores maiores com o aumento da razão de aquecimento); fatores estruturais (uma cura mais completa em valores mais elevados para a razão de aquecimento devido à presença de um maior número de grupos epóxi na reação), e fatores cinéticos (o aumento da razão de aquecimento aumenta a temperatura, que diminui o tempo de reação de cura).

Dados cinéticos dos sistemas de pré-impregnados também foram obtidos via estudo isotérmico (método de Málek $^{[16]}$ ) e encontram-se na Tabela 2.

Observa-se pela Tabela 2 que a ordem de reação do siste- ma de resina $F 161$ varia de 1,17 a 1,87 com média em 1,54 . $\mathrm{O}$ processo de gelificação deve ocorrer em valores de porcentagem de cura semelhantes, pois o processo de densificação de ligações cruzadas independe da temperatura de cura. Por outro lado, o processo de vitrificação depende do grau de conversão do sistema de resina. Quanto maior for a temperatura de cura de um sistema maior será o processo de vitrificação e, conseqüentemente, maior será a temperatura de transição vítrea do material em estudo.

Os sistemas de pré-impregnados de resina epóxi F584 e 8552 apresentam ordem de reação em torno de 2, indicando que a reação é bimolecular e depende da concentração dos reagentes, que são, para ambos os sistemas de pré-impregnados, a resina epóxi e o agente de cura, uma amina. Como são bem descritas na literatura ${ }^{[10-27]}$, as reações entre grupos epóxiamina podem ser catalisadas por impurezas e pelos grupos hidroxilas que são formados durante a reação. Por isto, o modelo autocatalítico foi utilizado para descrever os parâmetros cinéticos dos sistemas de epóxi em estudo. Mas para os sistemas de pré-impregnados estudados no presente trabalho, modelos cinéticos mais simples e de análises e cálculos mais rápidos, como o método dinâmico modelo de Borchardt-Daniels, pode ser utilizado para obter os parâmetros cinéticos desejados.

A equação de Borchardt-Daniels permite avaliar $E_{a}, \ln A$ e $n$. Porém, estes parâmetros cinéticos são influenciados pela razão de aquecimento. Os valores de $E_{a}$ e $\ln A$ obtidos pelo método dinâmico são ligeiramente superiores aos obtidos pelo método isotérmico. Por outro lado, a ordem de reação encon-

Tabela 2. Parâmetros cinéticos obtidos via método isotérmico dos sistemas de pré-impregnados $F 584$, 8552 e $F 161$.

\begin{tabular}{|c|c|c|c|c|c|c|}
\hline $\begin{array}{c}\text { Matriz do } \\
\text { pré-impregnado }\end{array}$ & $\begin{array}{l}\text { Isotermas } \\
\quad\left({ }^{\circ} \mathbf{C}\right)\end{array}$ & $n$ & $m$ & $\begin{array}{l}-\Delta H \\
(J / g)\end{array}$ & $\begin{array}{r}\ln A \\
\left(\mathrm{~s}^{-1}\right)\end{array}$ & $\begin{array}{c}E a \\
(k J / m o l)\end{array}$ \\
\hline \multirow{6}{*}{ F161 } & 120 & 0,87 & 0,30 & 42,1 & $21,6 \pm 0,1$ & \multirow[t]{6}{*}{$96,2 \pm 3,4$} \\
\hline & 125 & 0,67 & 0,68 & 45,4 & $16,8 \pm 0,1$ & \\
\hline & 130 & 0,62 & 0,75 & 41,1 & $19,7 \pm 0,1$ & \\
\hline & 135 & 0,78 & 0,98 & 40,9 & $18,8 \pm 0,1$ & \\
\hline & 140 & 0,98 & 0,74 & 40,7 & $20,3 \pm 0,1$ & \\
\hline & 145 & 1,3 & 0,57 & 46,6 & $21,1 \pm 0,1$ & \\
\hline \multirow{5}{*}{8552} & 155 & 1,15 & 0,86 & 113,9 & $9,4 \pm 0,1$ & \multirow[t]{5}{*}{$43,95 \pm 5,8$} \\
\hline & 160 & 1,18 & 0,82 & 114,1 & $9,6 \pm 0,1$ & \\
\hline & 165 & 1,19 & 0,81 & 115,6 & $9,4 \pm 0,1$ & \\
\hline & 170 & 1,13 & 0,88 & 116,7 & $9,7 \pm 0,1$ & \\
\hline & 175 & 1,16 & 0,85 & 117,3 & $9,6 \pm 0,1$ & \\
\hline \multirow{5}{*}{ F584 } & 150 & 1,80 & 0,23 & 114,9 & $8,4 \pm 0,1$ & \multirow[t]{5}{*}{$60,9 \pm 7,4$} \\
\hline & 160 & 1,55 & 0,58 & 115,2 & $8,6 \pm 0,1$ & \\
\hline & 165 & 0,92 & 1,10 & 116,7 & $8,4 \pm 0,1$ & \\
\hline & 170 & 1,33 & 0,69 & 117,1 & $8,7 \pm 0,1$ & \\
\hline & 175 & 1,48 & 0,61 & 118,6 & $8,6 \pm 0,1$ & \\
\hline
\end{tabular}


trada pelo método dinâmico é menor que a encontrada pelo método isotérmico. Para os sistemas estudados, existe uma diferença na ordem de reação encontrada pelo método dinâmico e pelo método isotérmico de $15 \%$ para o pré-impregnado F584; de $45 \%$ para o sistema 8552 e de $36 \%$ para o pré-impregnado F161. Assim, para fins de comparação entre estudos de cinética de cura é importante que se determine qual método foi utilizado na obtenção dos parâmetros cinéticos.

Apesar do método isotérmico não fazer aproximações nos cálculos e representar com maior precisão a realidade cinética, é um método muito demorado em relação ao dinâmico, uma vez que necessita de no mínimo três isotermas para se obter os parâmetros cinéticos. Os dados cinéticos obtidos foram determinados para auxiliar o desenvolvimento de ciclos de cura otimizados de pré-impregnados que já possuem um tempo de armazenagem maior que 6 meses. Sendo assim, análises mais rápidas fornecem respostas mais ágeis para auxiliar na caracterização de pré-impregnados aeronáuticos. Apesar do método dinâmico fornecer resultados aproximados dos parâmetros cinéticos, no presente estudo, este método mostrou-se como uma ferramenta rápida e adequada, para descrever o comportamento cinético dos sistemas de pré-impregnados investigados.

\section{Investigação reológica}

A Figura 1 apresenta as curvas viscosimétricas do préimpregnado de fibra de carbono/epóxi após ser submetido às análises reológicas. Como pode ser observado, o aumento da temperatura reflete inicialmente na diminuição da viscosidade complexa $\left(\eta^{*}\right)$ até ser atingida uma temperatura a partir da qual há o início do processo de cura do pré-impregnado com a formação de uma rede tridimensional. Esta diminuição de viscosidade ocorre devido à destruição gradativa das forças de interação (van der Waals) existentes na resina ainda não curada, sendo que esta estrutura pode ainda ser destruída com o aumento da taxa de cisalhamento e facilmente recomposta quando esta velocidade diminui.

A partir da análise da Tabela 3 foi constatado que a região de viscosidade mínima ocorreu em temperaturas mais elevadas com o aumento da razão de aquecimento devido, provavelmente, à uma cinética de cura mais lenta ocasionada pelo aquecimento mais rápido da amostra analisada. Entretanto, o valor da viscosidade mínima diminuiu de forma significativa com o aumento da razão de aquecimento, devido às temperaturas mais elevadas favorecerem a quebra de ligações secundárias existentes na estrutura da matriz polimérica.

Durante a análise viscosimétrica verificou-se uma evolução significativa da viscosidade complexa após a passagem pela região de mínimo de viscosidade, caracterizando um aumento contínuo da componente elástica, devido à uma rede formada por partículas ou agregados interconectados por interações elásticas, caracterizando a passagem de um sistema Newtoniano a um sistema viscoelástico. Durante a polimerização, à medida que a reação ocorre, as cadeias do polímero crescem, depois se ramificam e em função da densidade de ligações cruzadas, sua flexibilidade e mobilidade diminuem.
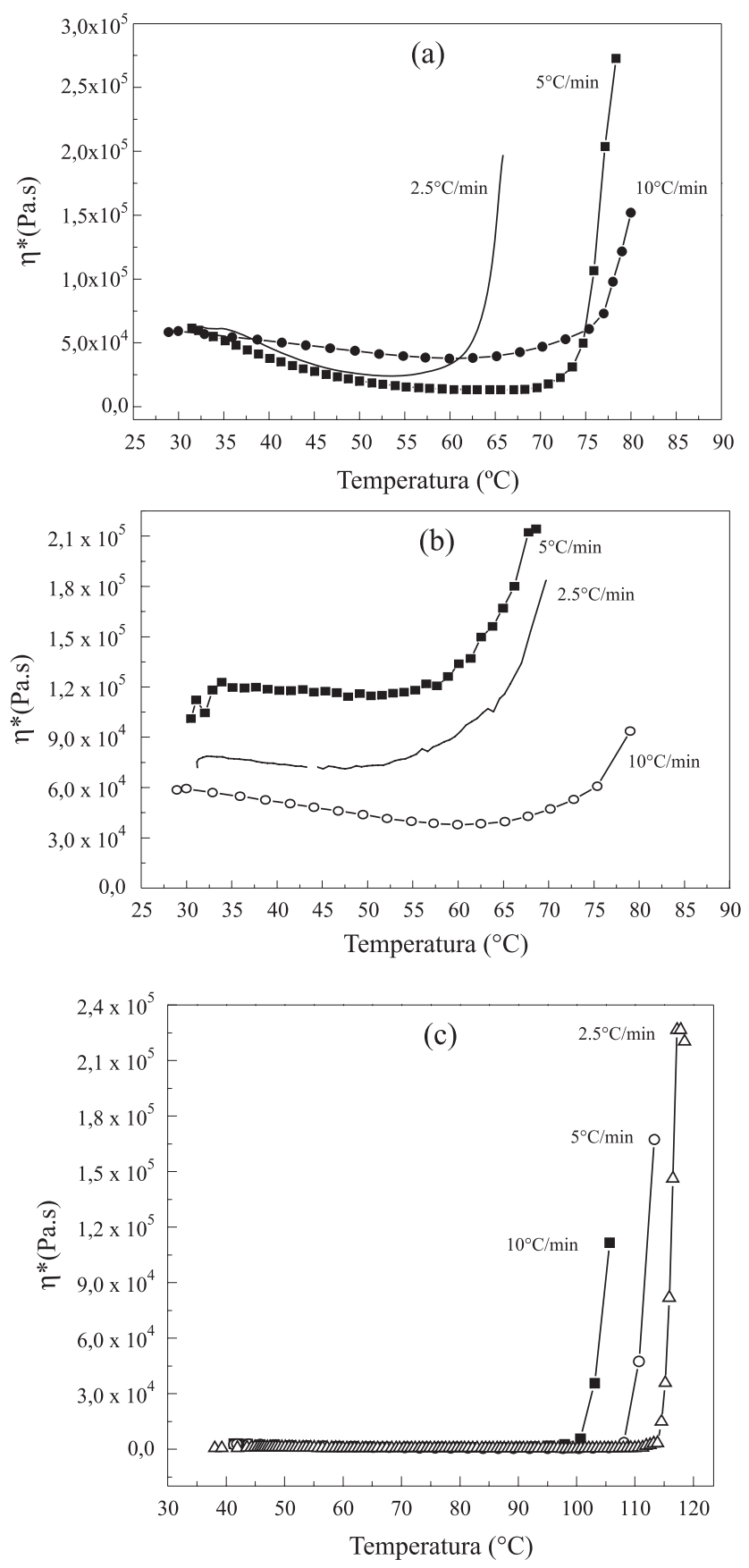

Figura 1. Evolução da viscosidade complexa dos pré-impregnados de carbono/epóxi $F 584$ (a), 8552 (b) e $F 161$ (c) com a temperatura, a diferentes razões de aquecimento.

A Tabela 3 evidencia também a influência da razão de aquecimento utilizada no começo da evolução da viscosidade complexa para a amostra analisada. Como pode ser observado, a evolução da viscosidade complexa, para diferentes razões de aquecimento de 2,$5 ; 5$ e $10{ }^{\circ} \mathrm{C} / \mathrm{min}$, começou a ocorrer na faixa de 60 a $80^{\circ} \mathrm{C}$ para os sistemas $F 584$ e 8552 , e de 105 a $115^{\circ} \mathrm{C}$, para o sistema $F 161$. Esta diferença significativa entre as temperaturas de início de evolução das curvas de viscosidade complexa com o aumento da razão de aquecimento, era esperado devido a um tempo maior de residência favorecer o início da cinética de cura do sistema analisado, 
Tabela 3. Variação da viscosidade complexa dos pré-impregnados F584, 8552 e F161 em função das razões de aquecimento.

\begin{tabular}{|c|c|c|c|c|c|c|c|c|c|}
\hline \multirow{2}{*}{$\begin{array}{c}\text { Razão de aquecimento } \\
\left({ }^{\circ} \mathrm{C} / \mathrm{min}\right)\end{array}$} & \multicolumn{3}{|c|}{ F584 } & \multicolumn{3}{|c|}{8552} & \multicolumn{3}{|c|}{ F161 } \\
\hline & $\underset{(\text { Pa.s })}{\eta_{\text {míma }}}$ & $\begin{array}{c}\mathbf{T}_{\eta \text { mínima }} \\
\left({ }^{\circ} \mathbf{C}\right)\end{array}$ & $\begin{array}{l}\mathbf{T}_{\text {gel }} \\
\left({ }^{\circ} \mathbf{C}\right)\end{array}$ & $\begin{array}{c}\eta_{\text {mínima }} \\
(\text { Pa.s })\end{array}$ & $\mathbf{T}_{\eta \text { mínima }}$ & $\begin{array}{l}\mathbf{T}_{\text {gel }} \\
\left({ }^{\circ} \mathbf{C}\right)\end{array}$ & $\underset{(\mathbf{P a} . \mathbf{s})}{\eta_{\text {míma }}}$ & $\mathbf{T}_{\eta \text { mínima }}$ & $\begin{array}{l}\mathbf{T}_{\text {gel }} \\
\left({ }^{\circ} \mathbf{C}\right)\end{array}$ \\
\hline 2,5 & 24 & 55 & 63 & 72 & 45 & 62 & 58 & 58 & 114 \\
\hline 5,0 & 15 & 58 & 73 & 113 & 47 & 63 & 241 & 60 & 107 \\
\hline 10 & 38 & 63 & 75 & 7 & 60 & 74 & 319 & 70 & 99 \\
\hline
\end{tabular}

ocasionando uma cura mais homogênea e controlada quando comparado com elevadas razões de aquecimento.

O aumento gradativo da viscosidade observado no início da análise deve-se à uma lenta reestruturação do sistema. Entretanto, com a simples observação da evolução da viscosidade complexa não é possível determinar o tempo de gel com precisão. No acompanhamento viscoelástico, o tempo de gel pode ainda ser obtido por dois outros métodos: pelo cruzamento entre G' (módulo de armazenamento) com G" (módulo de perda) ou pela variação do $\tan \delta$ (amortecimento), na qual a temperatura de cura começa a ocorrer para valores de $\tan \delta$ igual a $1\left(\tan \delta=\mathrm{G}^{\prime} / \mathrm{G}^{\prime}\right)$.

Devido aos compósitos poliméricos serem materiais viscoelásticos (ou seja, possuem uma resposta composta de uma parte elástica e outra viscosa) e assumindo-se que esses materiais foram submetidos à uma deformação oscilatória de cisalhamento de pequena amplitude ( $1 \mathrm{rad} / \mathrm{s})$, as tensões resultantes oscilarão com a mesma freqüência $\mathrm{w}$, mas não estarão em fase com a deformação, exibindo, então, um comportamento viscoelástico linear. Assim, G' está associado à componente em fase com a deformação, ou seja, está associado à contribuição elástica ou armazenamento de energia em cada ciclo e G" está associado à componente fora de fase.

Desta forma, a Tabela 4 apresenta os valores do cruzamento de G' e G' e a temperatura de gel obtida a partir deste valor para as amostras de pré-impregnados de fibra de carbono/epóxi analisadas a diferentes razões de aquecimento $\left(2,5^{\circ} \mathrm{C} / \mathrm{min} ; 5^{\circ} \mathrm{C} / \mathrm{min}\right.$ e $\left.10^{\circ} \mathrm{C} / \mathrm{min}\right)$. Todas as curvas foram obtidas a baixa freqüência $(1,0 \mathrm{rad} / \mathrm{s})$ e desta forma G' permanece inferior a G', confirmando que as características de um sólido elástico não são atingidas antes da região de gel. A partir desta tabela é também observado que os valores encontrados para a temperatura de gelificação aumentaram com o aumento da razão de aquecimento, confirmando os resul-

Tabela 4. Parâmetros reológicos dos sistemas estudados em função da razão de aquecimento.

\begin{tabular}{ccccccc}
\hline & \multicolumn{2}{c}{ F584 } & \multicolumn{2}{c}{$\mathbf{8 5 5 2}$} & \multicolumn{2}{c}{ F161 } \\
\cline { 2 - 7 } $\begin{array}{c}\text { Razão } \\
\left({ }^{\circ} \mathbf{C} / \mathbf{m i n}\right)\end{array}$ & $\begin{array}{c}\mathbf{G}^{\prime}=\mathbf{G} " \\
(\mathbf{M P a})\end{array}$ & $\begin{array}{c}\mathbf{T}_{\mathrm{gel}} \\
\left({ }^{\circ} \mathbf{C}\right)\end{array}$ & $\begin{array}{c}\mathbf{G}^{\prime}=\mathbf{G}^{\prime \prime} \\
(\mathbf{M P a})\end{array}$ & $\begin{array}{c}\mathbf{T}_{\mathrm{gel}} \\
\left({ }^{\circ} \mathbf{C}\right)\end{array}$ & $\begin{array}{c}\mathbf{G}^{\prime}=\mathbf{G}^{\prime \prime} \\
(\mathbf{M P a})\end{array}$ & $\begin{array}{c}\mathbf{T}_{\mathrm{gel}} \\
\left({ }^{\circ} \mathbf{C}\right)\end{array}$ \\
\hline 2,5 & 98 & 67 & 141 & 71 & 63972 & 116 \\
5,0 & 121 & 76 & 413 & 78 & 53650 & 111 \\
10 & 179 & 87 & 180 & 87 & 45467 & 103 \\
\hline
\end{tabular}

tados obtidos via análise viscosimétrica. Entretanto, os valores de Tgel obtidos via cruzamento de G' com G' foram ligeiramente superiores aos encontrados via análise viscosimétrica ( $\sim 5 \%$ maiores).

Com base nas informações dinâmicas obtidas via análises reológicas, pode-se concluir que a razão de aquecimento mais adequada para a obtenção de um compósito estrutural é a de $2,5^{\circ} \mathrm{C} / \mathrm{min}$, devido à esta razão de aquecimento proporcionar uma cura a uma temperatura mais baixa quando comparada a $5{ }^{\circ} \mathrm{C} / \mathrm{min}$ e $10^{\circ} \mathrm{C} / \mathrm{min}$, proporcionando uma polimerização mais homogênea e controlada.

Entretanto, é muito importante que se aplique pressão abaixo da temperatura de gel, a fim de garantir uma molhabilidade adequada das fibras dos compósitos. A temperatura ótima pode ser determinada com base em estudos reológicos isotérmicos. Sendo assim, isotermas entre $100{ }^{\circ} \mathrm{C}$, $110^{\circ} \mathrm{C}$ e $120^{\circ} \mathrm{C}$ foram realizadas para se obter o tempo de gel dos sistemas estudados. Isotermas abaixo de $100^{\circ} \mathrm{C}$ foram realizadas, mas devido ao tempo muito grande de análise (superior a 1 hora) não foram incluídas no trabalho, uma vez que se tornariam inviáveis de serem aplicadas em um ciclo de cura em autoclave. A Tabela 5 resume os tempos de gel obtidos para cada sistema.

A Tabela 5 mostra que as amostras dos sistemas de resina submetidos a temperaturas de $110^{\circ} \mathrm{C} \mathrm{e} 120^{\circ} \mathrm{C}$ apresentaram uma cura muito acelerada em relação às amostras ensaiadas a $100^{\circ} \mathrm{C}$. Assim, o tempo de gel escolhido para o ciclo de cura foi o de $100^{\circ} \mathrm{C}$ por permitir uma cura mais lenta e controlada evitando a formação de vazios e defeitos no processo de consolidação dos compósitos.

\section{Ciclos de cura}

A etapa de cura da matriz é um dos passos mais importantes no processamento de compósitos poliméricos. A qualidade do produto final é controlada em grande extensão pelo

Tabela 5. Tempos de gel para os pré-impregnados em estudo.

\begin{tabular}{cccc}
\hline $\begin{array}{c}\text { Temperatura } \\
\left({ }^{\circ} \mathbf{C}\right)\end{array}$ & $\begin{array}{c}\text { F584 } \\
\text { Tempo de gel } \\
(\mathbf{s})\end{array}$ & $\begin{array}{c}\mathbf{8 5 5 2} \\
\text { Tempo de gel } \\
(\mathbf{s})\end{array}$ & $\begin{array}{c}\text { F161 } \\
\text { Tempo de gel } \\
(\mathbf{s})\end{array}$ \\
\hline 100 & 121 & 124 & 135 \\
110 & 18 & 19 & 21 \\
120 & 15 & 17 & 18 \\
\hline
\end{tabular}


ciclo de cura (estabelece parâmetros de cura: tempo, temperatura, pressão e suas seqüências combinadas) utilizado no processamento do compósito polimérico.

$\mathrm{O}$ ciclo de cura utilizado pelo fabricante de artefatos em compósitos é estabelecido pelo fornecedor de pré-impregnados. Esses ciclos são propostos com base no conhecimento do comportamento reológico e da cinética de polimerização do sistema de resina usado como matriz impregnante. No entanto, muitas vezes, é necessário adequar o ciclo de cura sugerido pelo fornecedor às condições reais que se encontra o pré-impregnado. Esses pré-impregnados podem ficar armazenados, sob refrigeração de $18{ }^{\circ} \mathrm{C}$ negativos, por 6 meses, depois disto precisam ser revalidados e algumas alterações no ciclo de cura são necessárias para garantir um qualidade final adequada. Para isso, o conhecimento do estágio de cura real (comportamento reo-cinético) que se encontra o sistema de resina do pré-impregnado a ser processado é de grande importância na otimização de um ciclo de cura. Variações no grau de cura de um pré-impregnado, ou seja, avanços no grau de reticulação do sistema de resina impregnante em relação ao fornecido pelo fabricante do pré-impregnado, podem ocorrer em função das condições de transporte, armazenagem e manuseio deste material. O uso inadequado de um ciclo de cura pode acarretar na formação de regiões no laminado com distribuição heterogênea de resina/reforço (regiões ricas e pobres em resina), formação de vazios e microtrincas. A ocorrência destes defeitos pode comprometer o uso final de um componente, com o aumento de rejeitos com alto valor agregado.

Em sendo assim, a partir do conhecimento dos parâmetros cinéticos dos sistemas de pré-impregnados $F 584,8552$ e $F 161$ e da equação 4 , fez-se uma simulação matemática do grau de conversão da resina epóxi obtida em cada etapa do ciclo de cura.

$$
\alpha=1-\left[1-(1-n) \cdot \text { A.t. } \exp \left(-E_{a} / R T\right)\right]^{(1 /(1-n))}
$$

$\mathrm{O}$ ciclo de cura empregado industrialmente na autoclave e sugerido pelo fabricante de pré-impregnado compõe-se de 4 etapas, sendo: aquecimento de 30 a $116^{\circ} \mathrm{C}$ a $2,5^{\circ} \mathrm{C} / \mathrm{min}$; se-

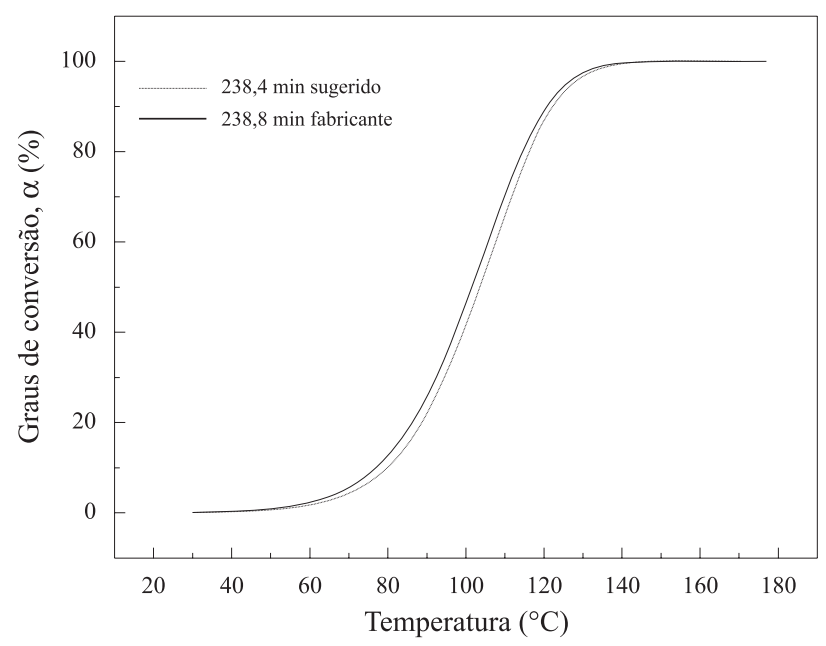

Figura 2. Grau de conversão total (médio) para os pré-impregnados de fibra de carbono/epóxi como função da temperatura na autoclave.

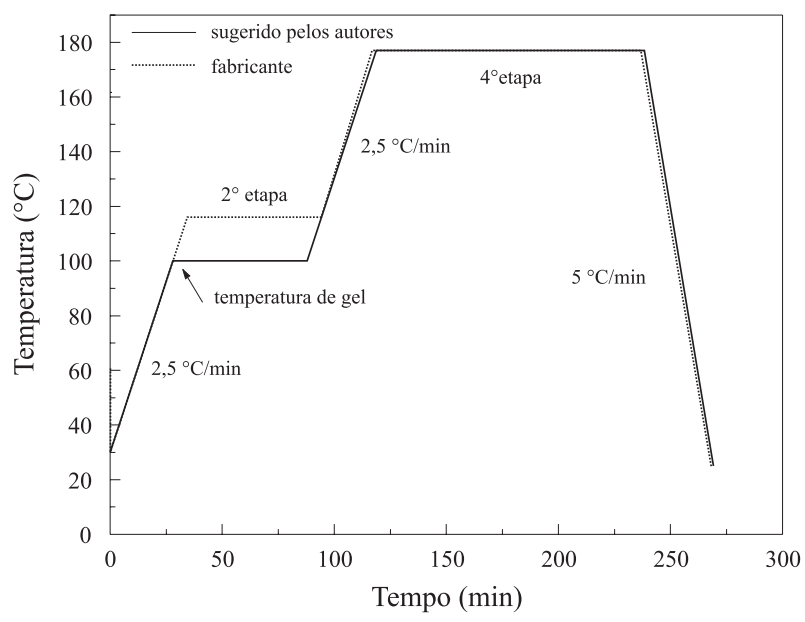

Figura 3. Ciclo de cura sugerido pelos autores para a cura dos pré-impregnados $F 584,8552$ e $F 161$

guido de uma isoterma a $116{ }^{\circ} \mathrm{C}$ por $60 \mathrm{~min}$; novo aquecimento de 116 a $177^{\circ} \mathrm{C}$ também a $2,5^{\circ} \mathrm{C} /$ min e por último uma isoterma a $177^{\circ} \mathrm{C}$ por no mínimo $120 \mathrm{~min}$.

Como o tempo de gel que fornece um cura controlada e homogênea para os três sistemas em estudo foi de $100^{\circ} \mathrm{C}$, esta temperatura foi escolhida como a temperatura onde se aplica pressão no lugar da temperatura de $116^{\circ} \mathrm{C}$ estabelecida pelo fabricante de pré-impregnados. Porém, para se atestar que esta temperatura de $100{ }^{\circ} \mathrm{C}$ confere um grau de cura final similar ao do fornecedor, a equação 4 foi utilizada para simular o grau de cura obtido a partir do ciclo de cura do fornecedor e do ciclo sugerido pelos autores (Figura 2).

Pode-se observar na Figura 2 que o grau de cura final médio obtido para os três sistemas de pré-impregnados curados com o ciclo de cura sugerido pelos autores é similar ao obtido com o ciclo de cura do fabricante. A proposição apresenta, ainda, a vantagem de reduzir a temperatura de $116^{\circ} \mathrm{C}$ para $100^{\circ} \mathrm{C}$, minimizando os gastos com a energia utilizada na autoclave, bem como o tempo de cura do processo total de compactação dos laminados de compósitos poliméricos. Com isto, estabeleceu-se um novo ciclo de cura destinado à compactação simultânea dos pré-impregnados de resina epóxi F584, 8552 e F161 (Figura 3).

\section{Conclusão}

A cura de uma matriz polimérica é considerada como sendo a chave para a fabricação de compósitos poliméricos avançados a partir de sistemas pré-impregnados, no qual a matriz encontra-se em um estágio intermediário de cura. A qualidade final do produto obtido pode ser controlada pelo estudo do ciclo de cura empregado associado aos parâmetros de processo, tais como: tempo, temperatura, pressão, assim como a seqüência na qual essas variáveis são combinadas.

A combinação de duas técnicas experimentais, análise térmica (DSC) e reologia, tem se mostrado muito eficiente na caracterização e no estabelecimento de cinéticas de cura de matrizes poliméricas. Os sistemas de pré-impregnados de resina epóxi $F 584$ e 8552 apresentam ordem de reação em 
torno de 2, indicando que a reação é bimolecular e depende da concentração dos dois reagentes. As reações entre grupos epóxi-amina podem ser catalisadas por impurezas e pelos grupos hidroxilas que são formados durante a reação. Por isto, o modelo autocatalítico foi utilizado para descrever os parâmetros cinéticos dos sistemas de epóxi. Mas para os sistemas de pré-impregnados estudados no presente trabalho, modelos cinéticos mais simples e de análises e cálculos mais rápidos, como o método dinâmico - modelo de BorchardtDaniels, podem ser utilizados para obter os parâmetros cinéticos desejados. A equação de Borchardt-Daniels permite avaliar tanto $E_{a}, \ln A$ e $n$. Porém, estes parâmetros cinéticos são influenciados pela razão de aquecimento. Os valores de $E_{a}$ e $\ln A$ obtidos pelo método dinâmico são ligeiramente maiores que os obtidos pelo método isotérmico. Entretanto, no presente estudo, o método dinâmico mostrou-se como uma ferramenta rápida, aproximada, mas adequada, para descrever o comportamento cinético dos sistemas de pré-impregnados investigados.

Com base nos parâmetros reológicos, a temperatura de início da cura, a ser utilizada no ciclo de cura, foi definida como $100^{\circ} \mathrm{C}$, devido à esta temperatura permitir uma cura mais controlada, com uma lenta reestruturação do sistema. Assim, a partir do conhecimento da cinética de cura e dos parâmetros reológicos dos sistemas de pré-impregnados estudados foi possível avaliar o ciclo de cura sugerido pelo fabricante, bem como estabelecer uma metodologia para otimizar o processamento do compósito em autoclave.

\section{Agradecimentos}

Os autores agradecem à FAPESP (02/01808-7; 02/012883) e ao $\mathrm{CNPq}$ (300599/96) pelo suporte financeiro.

\section{Referências Bibliográficas}

1. Segal, L. C. - SAMPE Journal, 32, p.12 (1996).

2. Cândido, G. M.; Almeida, S. F. M. \& Rezende, M. C. Polímeros: Ciência e Tecnologia, 10, p.31 (2000).

3. Hergenrother, P. M. - SAMPE Journal, 36, p.30 (2000).

4. Morgan, R. J. - SAMPE Journal, 37, p.102 (2001).

5. Costa, M. L.; Almeida, S. F. M. \& Rezende, M. C. Composite Science and Technology, 61, p.2101 (2001).

6. Strong, A. B. - "Fundamentals of composites manufacturing: materials, methods, and applications". Michigan: Society of Manufacturing Engineers, USA (1989).

7. Rashkovan, I.A. - Composites Science and Technology, 57, p.1017 (1997).
8. Abaris Training, - "Advanced Composite Structures: Fabrication and Damage Repair", Reno NV, EUA, p.205 (1998).

9. Costa, M.L. - "Efeito do conteúdo de vazios na resistência mecânica de compósitos avançados de carbono/epóxi e carbono/bismaleimida". Tese de Doutorado, ITA, São José dos Campos, SP, Brasil (2002).

10. Breitigam, W.V.; Bauer, R.S. \& May, C.A. - Chemtech, 23, p.38 (1993).

11. Varley, R.J.; Hodgkin, J.H.; Hawthorne, D.G.; Simon, G.P. \& McCulloch, D. -Polymer, 41, p.3425 (2000).

12. Wisanrakkit, G. \& Gilham J.K. - Journal of Applied Polymer Science, 41, p.2885 (1990).

13. Jenninger, W.; Schawe, J.E.K. \& Alig, I. - Polymer, 41, p.1577 (2000).

14. Lee, C.L. \& Wei, K.H - Journal of Applied Polymer Science, 77, p.3149 (2000).

15. Phillips, R.; Glauser, T. \& Manson, J.A.E.- Polymer Composites , 18, p.500 (1997).

16. Malek, J. - Thermochimica Acta; 200, p.257 (1992).

17. Montserrat, S.; Flaque, C.; Pages, P. \& Malek, J. - Journal of Applied Polymer Science; 56, p.1413 (1995).

18. Laza, J.M.; Julian, C.A.; Larrauri, E.; Rodriguez, M. \& Leon, L.M.- Polymer, 40, p.35 (1998).

19. Wingard, C.D. - Thermochimica Acta, 357-358, p.293 (2000).

20. Hayes, B.S.; Gilbert, E.N. \& Seferis, J.C.- Composites: Part A, 31, p.717 (2000).

21. Montserrat, S \& Cima, I. - Thermochimica Acta, 330, p.189 (1999).

22. Ellis, B. - "The kinetics of cure and network formation", in: B. Ellis (Ed.), Chemistry and Technology of Epoxy Resins, Chapter 3, Blakie, London (1993).

23. Gillham, J.K. - Polymer Eng. Sci, 26, p. 1429 (1986).

24. Montserrat, S. - J. Appl. Polym. Sci, 42, p.545 (1992).

25. Costa, M.L.; Botelho, E.C.; Paiva, J.M.F. \& Rezende, M.C. - Polímeros: Ciência e Tecnologia, 13, p.188 (2003).

26. Costa, M.L.; Pardini, L.C. \& Rezende, M.C. - Polímeros: Ciência e Tecnologia. Abr/Jun, n. 2, p.37 (1999).

27. Costa, M.L.; Botelho, E.C. \& Rezende, M.C. - Journal of Thermal Analyses and Calorimetric (2004) (in press).

Enviado: 25/11/04

Reenviado: $15 / 04 / 05$

Aprovado: $30 / 04 / 05$ 